\title{
Modeling and Experiment for Oxygen Isotope Exchange over Copper-containing Mordenite
}

\author{
Mikalai A. Artsiusheuski ${ }^{a, b}$, Jeroen A. van Bokhoven ${ }^{a, b}$, Vitaly L. Sushkevich ${ }^{b, *}$ \\ ${ }^{a}$ Laboratory for Catalysis and Sustainable Chemistry, Paul Scherrer Institut, 5232 \\ Villigen PSI, Switzerland \\ ${ }^{b}$ Institute for Chemistry and Bioengineering, ETH Zurich, Vladimir-Prelog-Weg 1, 8093 \\ Zurich, Switzerland
}

\section{Isotope exchange theory}

Taking into account the symmetry interconnection of the rate constants for the direct and reverse reactions for the particular type of isotope exchange, kinetics of exchange can be described quantitatively. It was previously shown ${ }^{1}$ that the following differential equations describe single-atom isotope exchange:

$$
\begin{aligned}
& \frac{\partial a}{\partial t}=-\frac{1}{2} \cdot C(O)_{\text {solid }} \cdot k_{1}\left(\alpha-a_{s}\right) \\
& \frac{\partial f_{34}}{\partial t}=C(O)_{\text {solid }} \cdot k_{1} \cdot\left(\alpha\left(1-a_{s}\right)+a_{s}(1-\alpha)-f_{34}\right) \\
& \frac{\partial a_{s}}{\partial t}=C\left(O_{2}\right)_{\text {gas }} \cdot k_{1}\left(\alpha-a_{s}\right)
\end{aligned}
$$

The same approach for two-atom exchange results in the following set of equations:

$$
\begin{aligned}
& \frac{\partial a}{\partial t}=-\frac{1}{2} \cdot\left(C(O)_{\text {solid }}\right)^{2} \cdot k_{2}\left(\alpha-a_{s}\right) \\
& \frac{\partial f_{34}}{\partial t}=\left(C(O)_{\text {solid }}\right)^{2} \cdot k_{2} \cdot\left(2 a_{s}\left(1-a_{s}\right)-f_{34}\right) \\
& \frac{\partial a_{s}}{\partial t}=C(O)_{\text {solid }} \cdot C\left(O_{2}\right)_{\text {gas }} \cdot k_{2}\left(\alpha-a_{s}\right)
\end{aligned}
$$


where $C(\mathrm{O})_{\text {solid }}$ and $C\left(\mathrm{O}_{2}\right)_{\text {gas }}$ are the concentration of exchangeable atoms in solid $\left(\mathrm{mol} \cdot \mathrm{l}^{-1}\right)$ and oxygen molecules concentration in the gas phase $\left(\mathrm{mol} \cdot \mathrm{l}^{-1}\right)$, respectively, and $\mathrm{k}_{1}\left(1 \cdot \mathrm{mol}^{-1} \cdot \mathrm{s}^{-1}\right)$ and $\mathrm{k}_{2}\left(\mathrm{l}^{2} \cdot \mathrm{mol}^{-2} \cdot \mathrm{s}^{-1}\right)$ are constants of single-atom and two-atom exchange. Combining equations above, we can describe kinetics of the oxygen isotope exchange in the systems, where simultaneous single- and two-atom exchange take place.

\section{Estimation of the effect of diffusion}

For the numerical modelling of the molecular oxygen interpartical diffusion Wolfram Mathematica 12 software $^{2}$ was used. Zeolite MOR has the pore system comprising 1D 12-MR channels with 8-MR pockets. We assumed that the diffusion along the channels has main impact, thus, the overall diffusion process may be considered as 1D diffusion and described by Fick's law:

$$
\frac{\partial C}{\partial t}=D \frac{\partial^{2} C}{\partial r^{2}}
$$

with initial and boundary conditions, assuming i) the initial concentration inside the particle is equal to zero, ii) the concentration on the surface of the particle is $\mathrm{C}_{0}$ and does not change with time, iii) there is no diffusion flux in the center of the particle:

$t=0: \quad C(r)=0$

$R=R_{0}: C(t)=C_{0}$

$R=0: \frac{\partial C}{\partial \mathrm{r}}=0$

The numerical solution of this system provides concentration profiles inside the zeolite particle as a function of time with various diffusion coefficients. Obtained profiles are presented in Figure S1. 


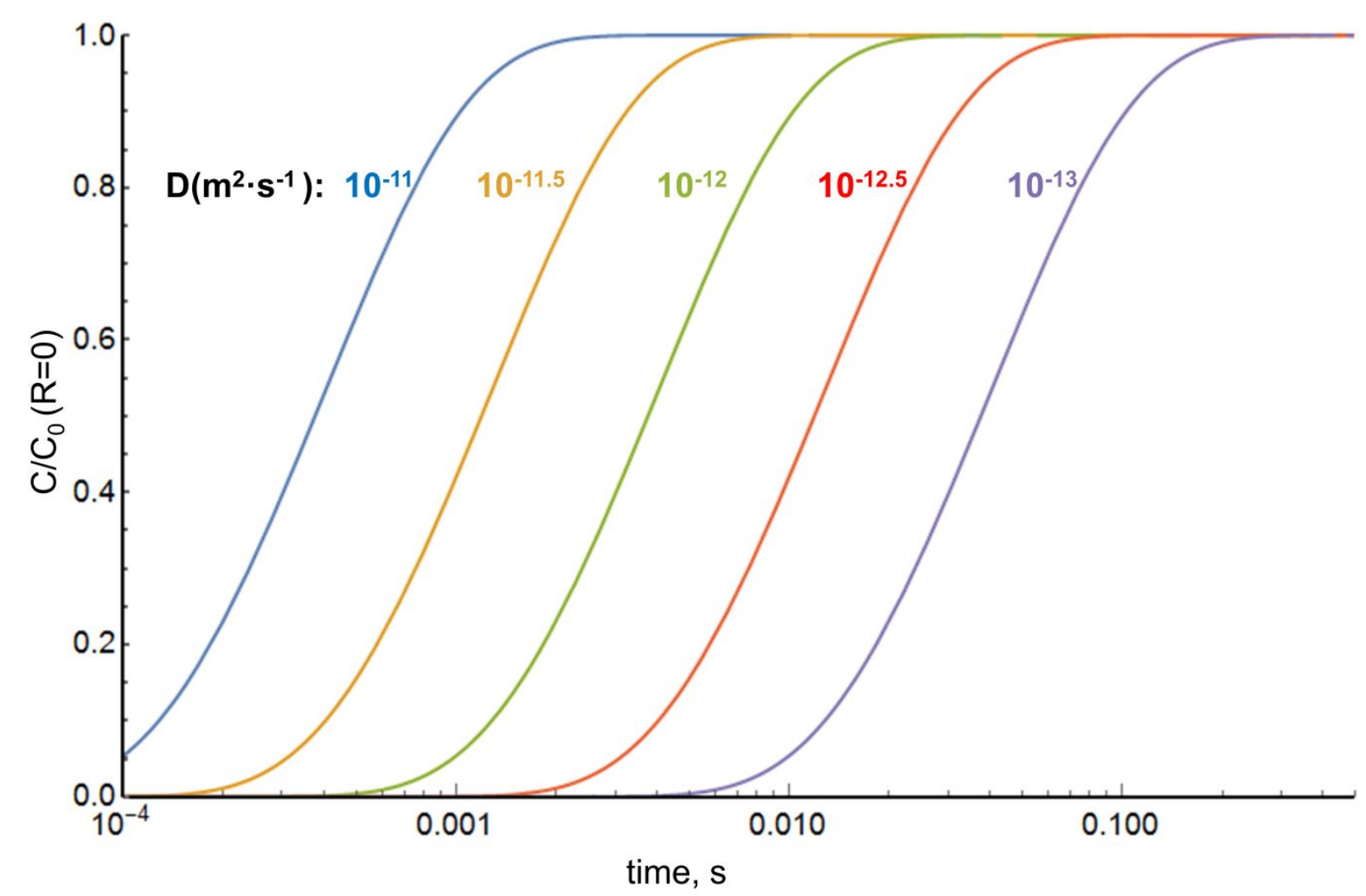

Figure S1. Relative concentration of diffusing molecules in the center of $200 \mathrm{~nm}$ particle for different diffusion coefficients.

The concentration gradients inside the particle are negligible after 0.1 second after the start of the diffusion even for diffusion coefficient an order of magnitude lower than reported for oxygen in zeolites 3 .

\section{Characterization of copper-containing mordenite}

The ion-exchange to copper leads to copper loading 4.35 wt.\%, which corresponds to $\mathrm{Cu} / \mathrm{Al}$ ratio equal to 0.38 .

\section{Isotope exchange over copper-containing mordenite}

Initial data for the time dependence of the signals with $\mathrm{m} / \mathrm{z}=32,34,36$ and 40 , and the behavior of the sum value of signals corresponding to all oxygen isotopologues are shown in Figure S2. The constant level of the sum value for all experimental temperature indicates that during the exchange the total concentration of oxygen molecules in the gas phase remains constant. The stepwise shape of the signal $\mathrm{m} / \mathrm{z}=40$ due to Ar confirms the proximity of the setup to a plug-flow reactor 

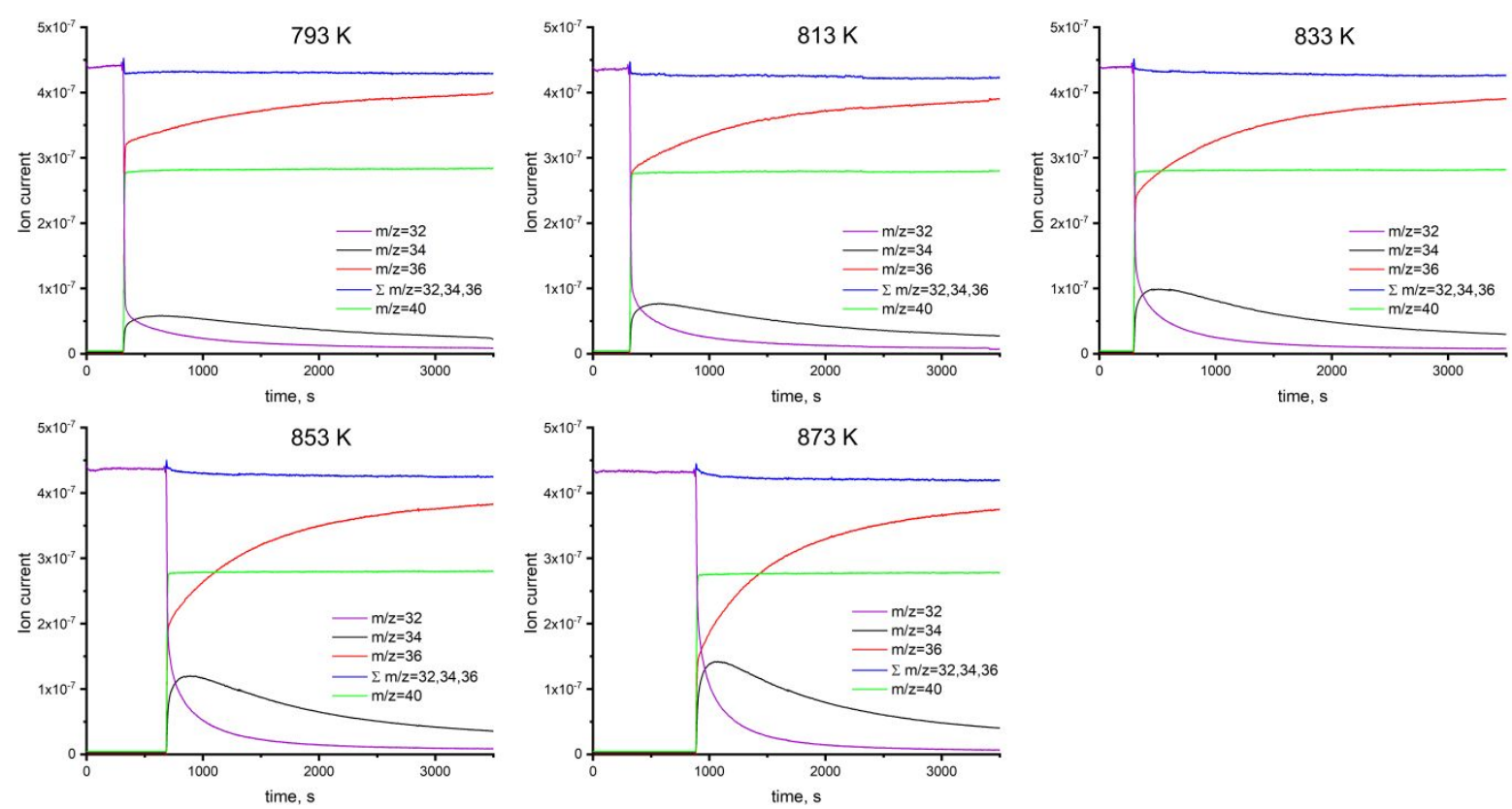

Figure S2. Time dependence of the signals with $\mathrm{m} / \mathrm{z}=32,34,36$ and 40 together with the sum value of signals corresponding to all oxygen isotopologues during the isotopic exchange.

Figures S3 a) and b) demonstrate fitting of the $\alpha$ and $f_{34}$, respectively, using a numerical model with only one type of exchangeable oxygen. Significant misfit was observed, therefore such a model cannot be used for accurate description of the oxygen isotopic exchange over copper-containing mordenite.
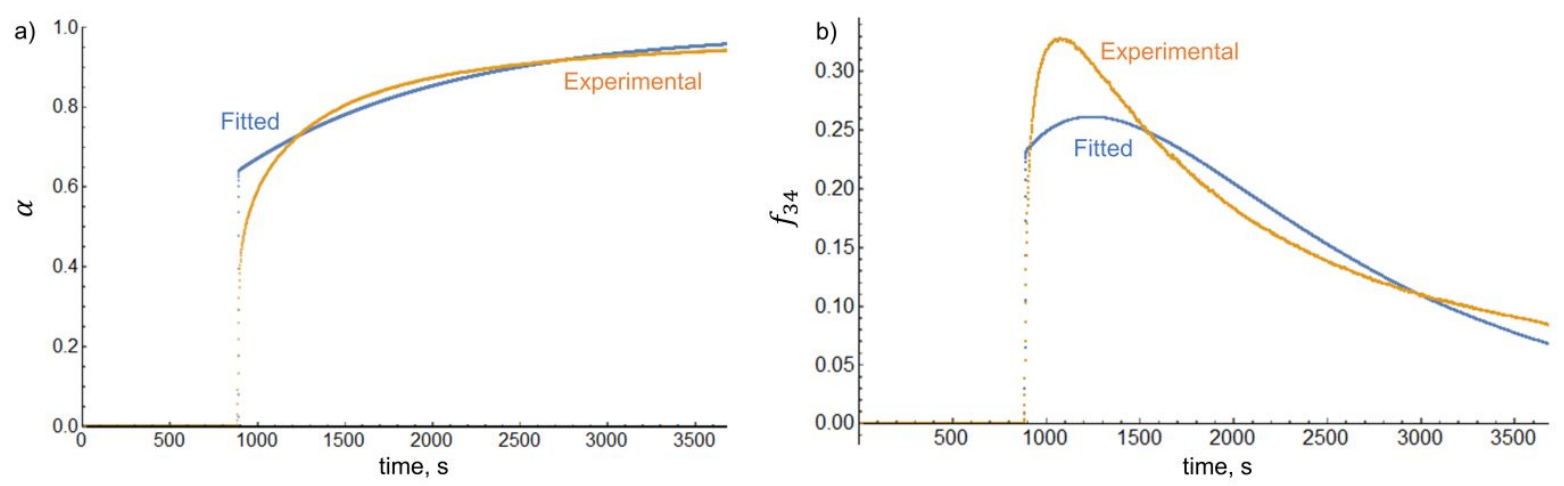

Figure S3. Experimental data points (orange color) and fitted curves (blue color) for parameters $\alpha$ (a) and $f_{34}$ (b) obtained at experimental temperature $873 \mathrm{~K}$. Numerical model with only one type of exchangeable oxygen was applied.

\section{Further increase the number of distinct exchangeable atoms in the kinetic model}

A priory, the kinetic model with three or even more kinetically different sources of exchangeable atoms can be developed. However, adding additional parameters, 
namely, concentration and kinetic constants corresponding to the third source, will increase number of the independent variables from 6 to 9, and, in turn, decrease the accuracy of determination of each variable. Moreover, placing of additional terms into PDE system may affect the stability of the solutions of the system, complicating the fitting procedure hence significantly decreasing the confidence of the results. Moreover, in some cases the global minimum can be even not determined numerically. Therefore, we believe that since the model accounting two distinct sources adequately describes the kinetics of exchange over copper-containing mordenite,there is no necessity to consoider three or more sites for CuMOR. However, the need of three sources cannot be excluded for other copper-exchanged zeolites or copper-containing materials.

\section{Model sensitivity and error estimation}

For all parameters, the deviation from the minimum point, which provides the best fit, leads to an increase of the NMRSD value i.e. to worse match of the simulated data to the experimental. This confirms that obtained values of the kinetic parameter may be considered as at least the local minimum point for the problem of functional minimization. In addition, such an analysis shows different fitting sensitivity to different parameters. For example, Figure S4 represents the effect of the relative deviation of each parameter on the NMRSD value for the experiment performed at 793 K. 


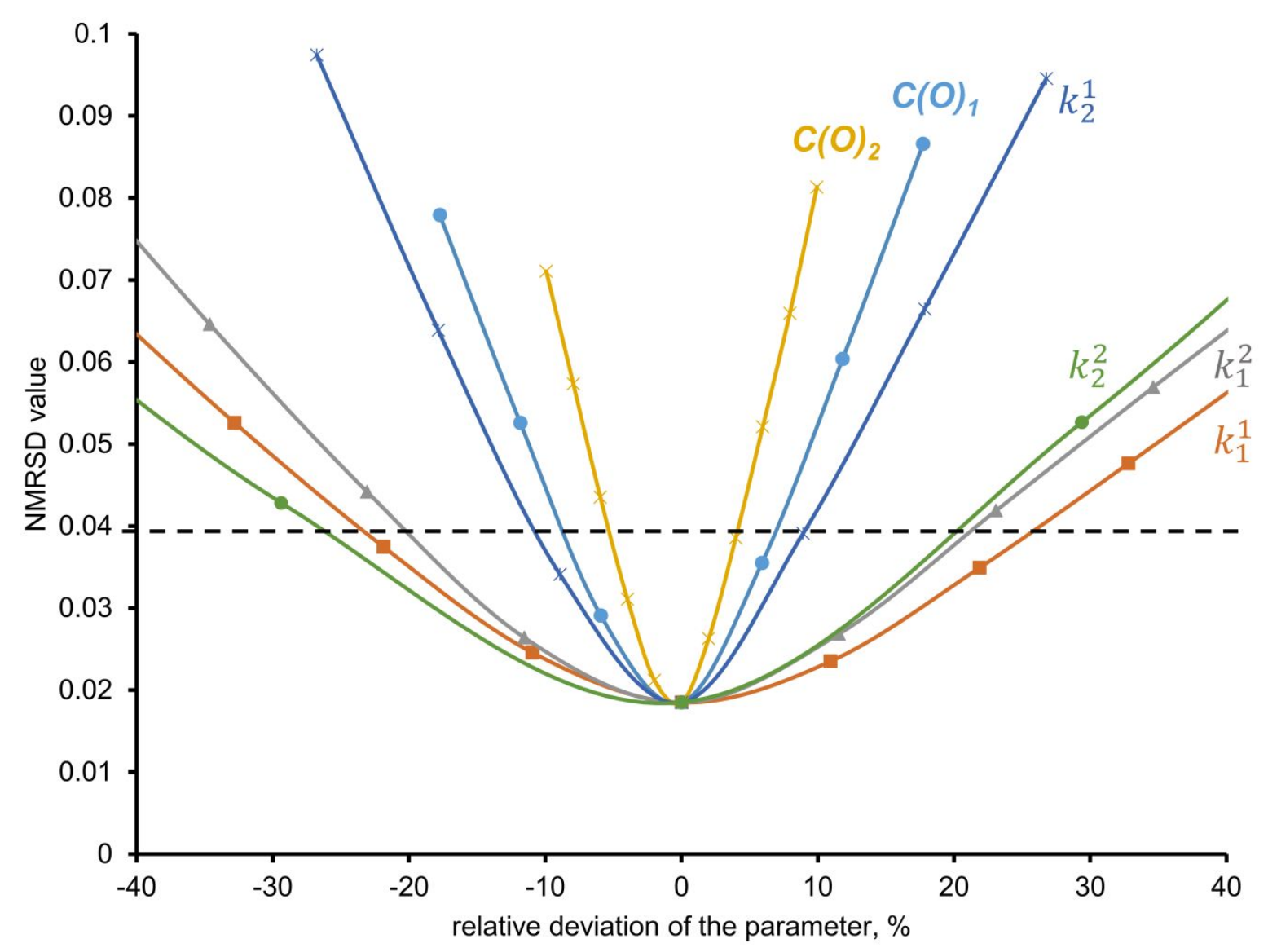

Figure S4. The effect of the relative deviation of each kinetic parameter on the NRMSD value. For the visualization, the dashed horizontal line shows the level corresponding to a value of the minimizing function two times more than that in the point of the best fit.

Thus, to achieve the same level of increase in NMRSD value, for example two times more than that in the point of the best fit (marked with dashed horizontal line on the Figure S4), the changes in parameter $C(O)_{2}$ should be around $5 \%$, while for $k_{1}^{1}$ it should be more than $20 \%$. Now, is we assume that used model fully accounts for all ongoing processes and all the differences between the theoretical and experimental curves are associated exclusively with experimental errors, we may estimate the error in the defined parameters as the deviation, which creates the increase in NMSRD value equal to that observed in the point of the best fit. The relative errors for the kinetic parameters estimated using described approach are presented in table S1. Comparison of the relative errors shows that the concentration of the sources is determined with 
higher precision compared to that for the kinetic constants. Within one source, the higher precision is observed for the kinetic constant which corresponds for prevailing type of exchange.

Table S1. Relative errors for kinetic parameters extracted from fitting of the experimental data for $793 \mathrm{~K}$.

\begin{tabular}{|c|c|c|c|c|c|c|}
\hline Parameter & $C(O)_{1}$ & $k_{1}^{1}$ & $k_{1}^{2}$ & $C(O)_{2}$ & $k_{2}^{1}$ & $k_{2}^{2}$ \\
\hline Lower limit & $-8 \%$ & $-22 \%$ & $-20 \%$ & $-5 \%$ & $-9 \%$ & $-25 \%$ \\
\hline Upper limit & $+9 \%$ & $+24 \%$ & $+17 \%$ & $+6 \%$ & $+10 \%$ & $+20 \%$ \\
\hline
\end{tabular}

\section{References}

(1) Muzykantov, V. S.; Popovskii, V. V; Boreskov, G. R. Kinetics of Isotope Exchange in a Molecular oxygen-Solid Oxide system. Kinet. i Katal. 1964, Vol: 4.

(2) Mathematica, version 12.0; software for technical computation; Wolfram Research: Champaign, IL, 2004.

(3) Sun, W.; Costa, C. A. V. Activated Diffusion of Permanent Gases in 4a Zeolite at Low Coverage. Chem. Eng. Sci. 1994, 49 (12), 1913-1923. https://doi.org/10.1016/00092509(94)80076-6.

(4) Kornic, S.; Baker, M. Nanoporous Zeolite Film Electrodes. Chem. Commun. 2002, 2 (16), 1700-1701. https://doi.org/10.1039/b204042e. 